\title{
Una variación anatómica: la desembocadura aberrante del conducto torácico
}

\author{
Elizabeth Peña, Janneth Zuñiga \\ Departamento de Morfología, Facultad de Salud, Universidad del Valle, Cali, Colombia
}

\begin{abstract}
En uno de los cadáveres disecados durante el primer semestre de 2006 en el anfiteatro del Departamento de Morfología de la Universidad del Valle en Cali (Colombia), se encontró una variación anatómica poco descrita en la literatura científica mundial. Se trata de la desembocadura aberrante del conducto torácico. Normalmente, este conducto linfático asciende en el tórax y se desvía hacia el lado izquierdo del cuello para desembocar en el confluente venoso yúgulosubclavio izquierdo. En este cadáver masculino de etnia mestiza, el conducto se desvió hacia el lado derecho del cuello y desembocaba en la vena yugular interna derecha. El trabajo describe el origen embrionario del conducto torácico y ofrece una posible explicación para la anomalía encontrada.
\end{abstract}

Palabras clave: variación (genética), conducto torácico, anomalías cardiovasculares, anomalías linfáticas, anomalías múltiples.

\section{An anatomical variation: the aberrant termination of the thoracic duct}

In a male cadaver dissected at the Department of Morphology, University del Valle, Cali (Colombia), a rarely described anatomical variation was found. It consisted of an aberrant termination or drainage of the thoracic lymph duct. Normally, this duct ascends in the thorax behind the esophagus, gradually diverges towards the left side of the neck and ends in the left jugulo-subclavian confluent--either in the internal jugular vein or in the subclavian vein. In the case of this cadaver, the thoracic duct diverged towards the right side of the neck to end in the right internal jugular vein. The present work describes the embryonic origin of the duct and offers a possible explanation for the anatomical variation encountered.

Key words: Variation (Genetics), thoracic duct, cardiovascular abnormalities, lymphatic abnormalities; abnormalities, multiple.

Existe actualmente un volumen muy significativo de publicaciones a nivel mundial en el campo de las variaciones anatómicas. ¿Qué importancia tienen éstas? Muchas pueden acompañarse de manifestaciones clínicas, como es el caso de la disfagia o dificultad para deglutir, que puede presentarse en caso de un doble arco o cayado aórtico que comprima al esófago $(1,2)$. En las intervenciones quirúrgicas, el conocimiento de

\footnotetext{
Correspondencia:

Elizabeth Peña, Ciencias Básicas Médicas, Departamento de Morfología, Facultad de Salud, Universidad del Valle, sede San Fernando, calle 4 B № 36-00, edificio 116, Cali, Colombia.

Teléfono: 518 5629; fax: 518 5627; celular: (317) 4289637

celipeto@univalle.edu.co
}

Recibido: 25/08/08; aceptado:20/02/09 las posibles variaciones anatómicas que puede tener un determinado órgano evita que sea lesionado con consecuencias probablemente irreversibles para el paciente. Tal es el caso del daño que puede sufrir un nervio laríngeo inferior no recurrente en el curso de una tiroidectomía (2). Fueron estas consideraciones las que llevaron a las autoras a publicar su hallazgo. Con el fin de brindar una mayor claridad sobre la variación encontrada, se presenta, en primera instancia, la descripción de la anatomía y la embriología normales $y$, desde esta última, se ofrece una posible explicación para lo hallado.

\section{Anatomía}

El conducto torácico tiene un calibre normal de 2 a $4 \mathrm{~mm}$, aproximadamente, y transporta entre 
$2 \mathrm{ml}$ y $10 \mathrm{ml}$ de linfa por hora (3). En la mayoría de las personas nace en el abdomen, en una dilatación donde confluyen los troncos linfáticos lumbares e intestinales, llamada cisterna del quilo. Esta cisterna se ubica entre la aorta abdominal y la cruz derecha del diafragma, por encima del nivel de la arteria renal derecha, por delante de los cuerpos vertebrales de LI y LII.

De la parte superior de la cisterna del quilo (cisterna de Pecket) emerge el conducto torácico, el cual pasa al tórax por el hiato aórtico del diafragma y se ubica por detrás del esófago. Continúa en un trayecto mediano por el mediastino posterior, situado por delante de los cuerpos de las vértebras torácicas, entre la aorta torácica a la izquierda y la vena ácigos a la derecha. Sube por el mediastino superior, paulatinamente se desvía hacia la izquierda, penetra al cuello y describe una curva convexa hacia arriba para terminar desembocando en el confluente yúgulo-subclavio izquierdo, bien sea en la vena yugular interna, en la subclavia o en la braquiocefálica (4).

\section{Embriología}

Desde el punto de vista embrionario, más tardíamente que el desarrollo del sistema cardiovascular, hacia la quinta semana, se forma en el embrión el sistema linfático, a partir de hendiduras del mesénquima. Aparecen en el embrión plexos capilares linfáticos que acompañan a los troncos venosos primarios $y$, en determinadas regiones, se fusionan y forman los sáculos linfáticos; a partir de estos sáculos, crecen hacia las diferentes regiones corporales los vasos linfáticos.

En el embrión se forman seis sáculos linfáticos primarios: dos yugulares en la unión de las venas subclavias con las venas cardinales anteriores, dos iliacos en la unión de las venas iliacas internas con las venas cardinales posteriores, y uno retroperitoneal cerca de la raíz del mesenterio y la cisterna del quilo por detrás del saco retroperitoneal. Solamente los sáculos yugulares adquieren una conexión permanente con el sistema venoso. Del rico plexo capilar que aparece en el tórax alrededor de la aorta y que comunica la cisterna del quilo con ambos sáculos yugulares, se derivan dos conductos torácicos unidos por numerosas anastomosis transversas. La parte caudal o inferior del conducto torácico izquierdo desaparece; la parte caudal o inferior del conducto torácico derecho, la anastomosis y la parte craneal o superior del conducto torácico izquierdo forman el conducto torácico que, por esta razón, desemboca en el ángulo o confluente yúgulo-subclavio izquierdo. De la parte craneal o superior del conducto torácico derecho se forma el conducto linfático derecho que desemboca, por esto, en el confluente yúgulo-subclavio derecho $(5,6)$ (figura 1).

\section{Presentación del caso}

Empleando el instrumental necesario para realizar la disección del cadáver y con las guías de disección suministradas por la docente de la asignatura, los estudiantes de Medicina de la Universidad del Valle de Cali que cursaron anatomía macroscópica en el año 2006, practicaron la disección a cuatro cadáveres de los que llegan al anfiteatro del Departamento de Morfología.

En uno de los cadáveres disecados, de sexo masculino y etnia mestiza, se encontraron algunas variaciones anatómicas de interés. Por la historia clínica se pudo conocer que el sujeto tenía 56 años; su causa de muerte fue una neumonía y no se informaba de ningún antecedente clínico de importancia.

Con el fin de tener el registro fotográfico de las variaciones anatómicas encontradas, la docente practicó una disección más detallada y minuciosa del lado derecho del cuello. Al estar aislando la arteria tiroidea inferior, se encontró con lo que parecía ser una vena que desembocaba en la vena yugular interna derecha. Haciendo el seguimiento de la estructura en sentido descendente, descubrió que se trataba del conducto torácico. En el cadáver en mención, la posición del conducto torácico en el mediastino posterior era igual a la tradicionalmente descrita, pero en el mediastino superior de manera paulatina se desviaba a la derecha, ascendía en el cuello y desembocaba en el confluente yúgulo-subclavio derecho. 


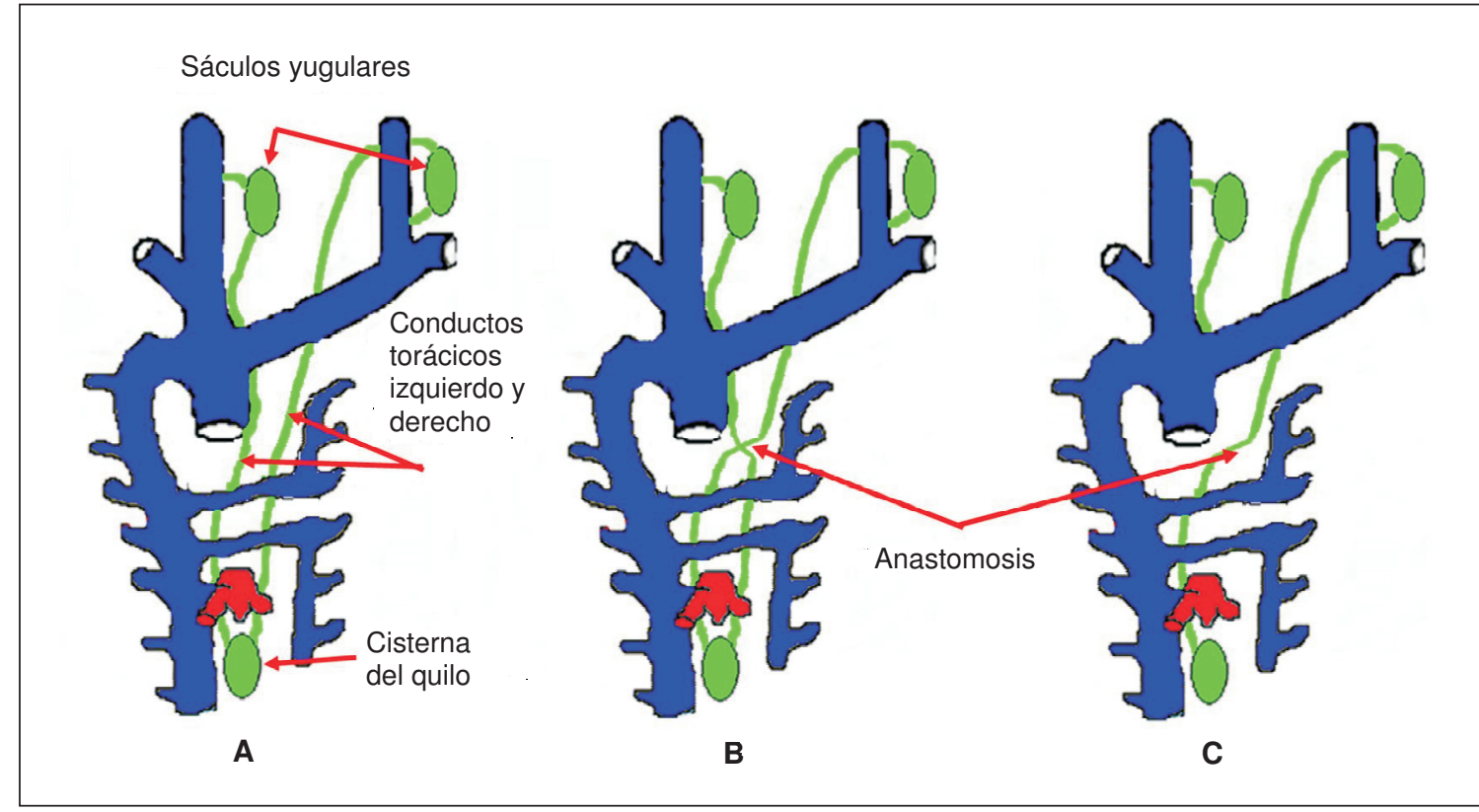

Figura 1. A) Se observan los conductos torácicos derecho e izquierdo que se conectan con los sáculos yugulares de cada lado, respectivamente. B) Muestra cómo estos conductos se unen mediante múltiples anastomosis transversas que aparecen a nivel torácico. C) Evidencia la reabsorción de la parte caudal o inferior del conducto torácico izquierdo.

En la figura 2 se aprecia el mediastino posterior de la cavidad torácica. A la izquierda aparece la aorta descendente y el esófago fue reclinado hacia la derecha para descubrir el conducto torácico. Éste se aprecia como un delgado cordón sinuoso de color blanquecino que cursa entre la aorta descendente y la vena ácigos, por delante de los cuerpos vertebrales. Se aprecia cómo va desviándose gradualmente hacia el lado derecho.

En la figura 3 se puede observar el conducto torácico en el cuello, donde alcanza a describir una ligera curva convexa hacia arriba para desembocar en la vena yugular interna derecha.

En la figura 4 se observa la vena yugular interna derecha y la desembocadura en ella del conducto torácico.

\section{Discusión}

En el presente caso se encontró un conducto torácico que, en lugar de dirigirse hacia la parte izquierda del cuello, lo hace hacia la derecha para desembocar en la vena yugular interna derecha. Éste suele llamarse conducto torácico derecho y el informe de esta variación en la literatura científica es escaso (2). Según van Pernis (7), en un estudio de 1.081 cadáveres encontró la variación en 61 (5,6\%) y Kausel (8) informa una incidencia de $2 \%$.

El trayecto aberrante o anómalo del conducto torácico encontrado en el cadáver se puede explicar por su formación embrionaria. Se puede deducir que en esta persona, en lugar de haberse reabsorbido la parte caudal o inferior del conducto torácico izquierdo, se reabsorbió esta parte del conducto torácico derecho, por lo cual el conducto torácico quedó formado por la parte caudal del conducto torácico izquierdo, la anastomosis y la parte craneal o superior del conducto torácico derecho que, por este hecho, se abre en la vena yugular interna derecha (figura 5).

Se podría suponer que, en este cadáver, la parte superior o craneal del conducto torácico izquierdo formó un conducto linfático izquierdo que se abriría en el confluente yúgulo-subclavio 


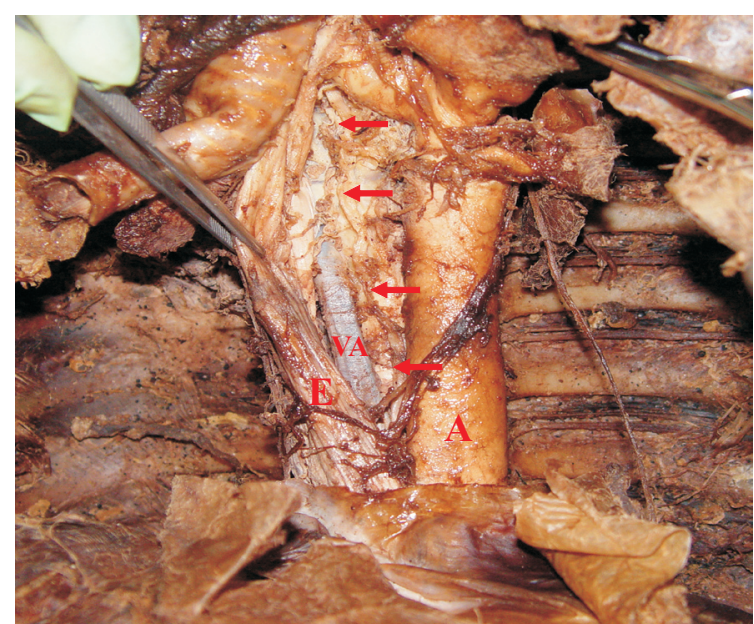

Figura 2. Se aprecia la aorta descendente (A), el esófago (E) y la vena ácigos (VA). El esófago fue reclinado hacia la derecha para descubrir el conducto torácico señalado con las flechas. En la parte superior se evidencia cómo empieza a desviarse hacia el lado derecho.

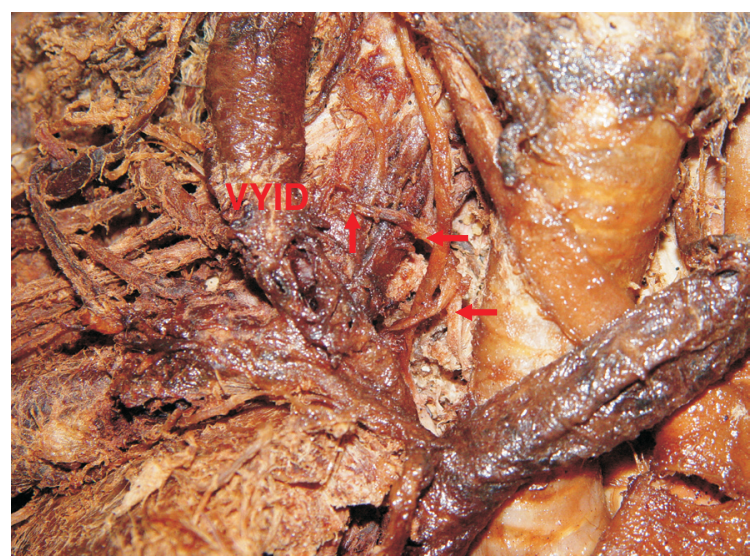

Figura 3. Se observa el conducto torácico en el cuello señalado por las flechas y se aprecia su desembocadura en la vena yugular interna derecha (VYID).

izquierdo. Sin embargo, en la disección no se encontró vestigio alguno de tal conducto, como tampoco se encontró un tronco yugular o un tronco subclavio abriéndose directamente en este confluente.

Con respecto a las variaciones del sistema linfático, Rodrigues et al. (9) reportan un estudio realizado en Brasil en el cual, de 34 cadáveres

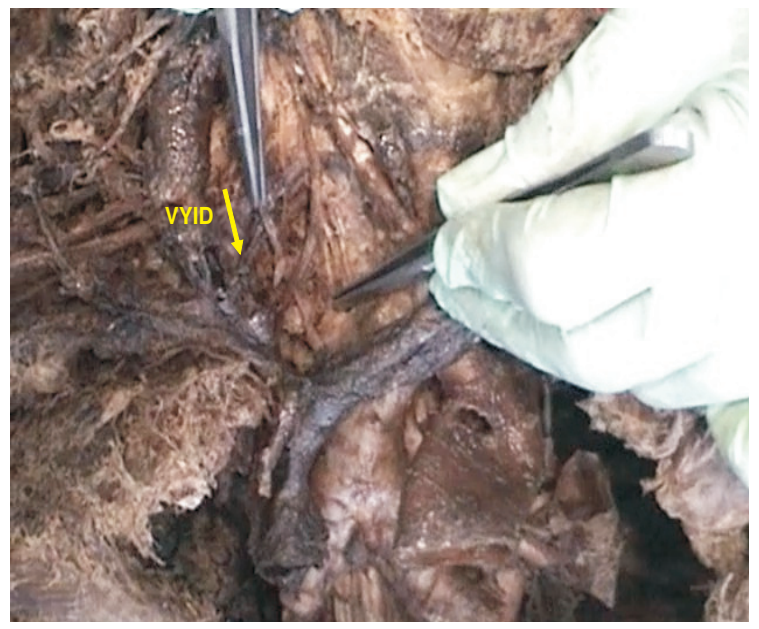

Figura 4. La arteria carótida común derecha ha sido ligeramente desplazada hacia la izquierda y con la pinza se ha levantado el conducto torácico señalado con la flecha. Se aprecia claramente la desembocadura de éste en la vena yugular interna derecha (VYID).

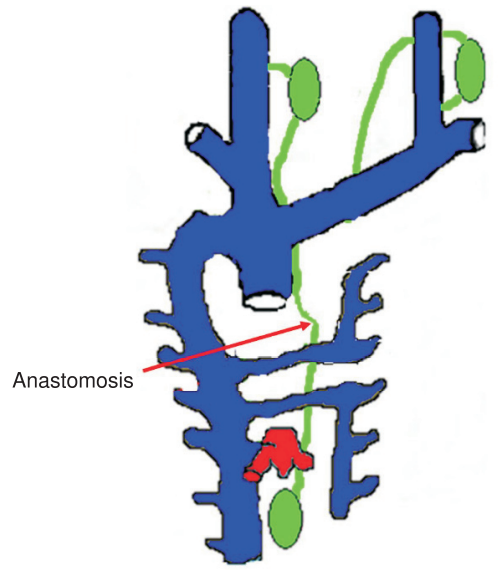

Figura 5. El esquema muestra la reabsorción de la parte caudal o inferior del conducto torácico derecho, por lo cual el conducto torácico derecho se forma de la parte inferior del conducto torácico izquierdo, la anastomosis y la parte craneal o superior del conducto torácico derecho.

disecados, encontraron $2(5,9 \%)$ con vasos linfáticos de apreciable calibre que no desembocaban en el conducto torácico sino directamente en la vena subclavia izquierda, cerca al ángulo yúgulo-subclavio. Ambos cadáveres presentaban conducto torácico con desembocadura normal en este ángulo. Los autores destacan que la lesión de estos vasos por su gran calibre puede producir una importante linforragia en la 
región, favoreciendo incluso la formación de un quilotórax. Igual podría ocurrir con un conducto torácico como el aquí informado, en el caso de una intervención quirúrgica del cuello donde no se tuviera en cuenta la posibilidad de esta variación anatómica.

Kinnaert (10) estudió la desembocadura del conducto torácico en 49 pacientes con insuficiencia renal crónica de carácter terminal, a quienes se les practicaron intervenciones quirúrgicas en el lado izquierdo del cuello y sólo en dos casos no las encontró; sin embargo, no se atreve a asumir que estos individuos presentaran conducto torácico derecho. En el estudio comparativo que realiza con datos de otros autores basados en disecciones de cadáveres, siempre que el conducto torácico no estaba presente en el lado izquierdo se trataba de conducto torácico derecho que desembocaba en la vena yugular interna derecha.

Como conclusión, se puede anotar que las variaciones anatómicas, aunque sean de baja incidencia en una determinada población, deben ser conocidas especialmente por los cirujanos, para evitar lesionar órganos importantes con repercusiones que pudieran ser irreversibles para los pacientes.

\section{Agradecimientos}

Las autoras agradecen al ingeniero Freddy Artunduaga por su valiosa asesoría técnica en la elaboración de las figuras.

\section{Conflictos de intereses}

Las autoras del presente trabajo declaramos que no existen conflictos de interés.

\section{Financiación}

Dado que el trabajo no es un proyecto de investigación no se requirió de una fuente de financiación ni interna ni externa. Los costos generados en la preparación del artículo fueron asumidos por las autoras.

\section{Referencias}

1. Márquez JP, García C. Caso radiológico pediátrico. Rev Chil Enf Respir. 2004;20:85-8.

2. Epstein DA, Debord JR. Abnormalities associated with aberrant right subclavian arteries - a case report. Vasc Endovascular Surg. 2002;36:297-303.

3. Solis JH, Sánchez C, Gutiérrez R. Variantes anatómicas del conducto torácico. Angiología. 1984;36:289-92.

4. Williams P, Warwick R. Gray anatomía. Tomo I. 36aㅡ ed. Barcelona: Salvat Editores S.A.; 1985.

5. Sadler TW. Embriología médica con orientación clínica. Novena edición. Madrid: Editorial Médica Panamericana; 2004.

6. van der Putte SC, van Limborgh J. The embryonic development of the main lymphatics in man. Acta Morphol Neerl Scand. 1980;18:323-35.

7. van Pernis PA. Variations of the thoracic duct. Surgery. 1949;26:806-9.

8. Kausel HW, Reeve TS, Stein AA, Alley RD, Stranahan A. Anatomic and pathologic studies of the thoracic duct. J Thorac Surg. 1957;34:631-42.

9. Rodrigues CFS, Wafae N, Olave E, Gabrielli C, Sgrott EA, Braga MT. Variaciones de las comunicaciones linfático-venosas en la fosa supraclavicular izquierda del hombre. Rev Chil Anat. 1997;15:175-9.

10. Kinnaert P. Anatomical variations of the cervical portion of the thoracic duct in man. J Anat. 1973;115:45-52. 